\title{
Micromagnetic Simulation of Domain Structure Transition in Ferromagnetic Nanospheres under Zero External Field
}

\author{
Dede Djuhana $^{1 *}$, Candra Kurniawan ${ }^{1,3}$, Dong-Hyun Kim² ${ }^{2}$ Agus Tri Widodo ${ }^{1}$ \\ ${ }^{1}$ Department of Physics, Faculty Mathematics and Natural Science (FMIPA), Universitas Indonesia, Kampus \\ UI Depok, Depok 16424, Indonesia \\ ${ }^{2}$ Department of Physics, Chungbuk National University, Cheongju 28644, South Korea \\ ${ }^{3}$ Research Center for Physics, Indonesian Institute of Sciences (LIPI), Puspiptek Office Area, Tangerang \\ Selatan 15314, Indonesia
}

\begin{abstract}
In this work, we investigated the domain structure transition in ferromagnetic nanospheres at the ground-state conditions under zero external magnetic field by micromagnetic simulation. Four basic ferromagnetic materials, nickel (Ni), permalloy (Py), iron (Fe), and cobalt (Co), with variation in diameters from 20 to $100 \mathrm{~nm}$ were modeled in the simulation. It was observed that a transition of domain structure occurs from a single-domain to a multi-domain structure at a specific diameter based on the magnetization energy profile. Interestingly, a vortexcore orientation in the multi-domain regime was related to the magnetocrystalline axis of the material, which first aligns with the hard-axis direction, and then changes to the easy-axis direction for low-anisotropy materials ( $\mathrm{Ni}, \mathrm{Py}$, and $\mathrm{Fe}$ ). In contrast, only hard-axis orientation exists for highanisotropy materials (Co). Furthermore, it is also observed that the transition of domain structure was related to the critical diameter. Below the critical diameter, a single-domain structure is exhibited in which the demagnetization energy was larger than the exchange energy. A multidomain structure emerged above the critical diameter where the exchange energy was larger than the demagnetization energy. The comparable values of critical diameter were also calculated based on the Kittel and Brown equations. The results of the critical diameter from the micromagnetic simulation agreed with the theoretical calculations. Therefore, an interpretation of these magnetization dynamics is an important step in the material selection for granular magnetic-based storage.
\end{abstract}

Keywords: Critical Diameter; Domain Structure; Micromagnetic; Multi-Domain; Single Domain

\section{Introduction}

The technological advancement of magnetic storage media was enhanced by an improved understanding of dynamic magnetization in ferromagnetic materials. Some potential application research of magnetic recordings, such as magnetic random-access memory (MRAM), magnetic logic, microwave oscillators, and magnetic nanosensors, has grown extensively in recent years (Udhiarto et al., 2014; Sun et al., 2015; Joshi, 2016; Bhatti et al., 2017; Sbiaa and Piramanayagam, 2017). Magnetic storage technologies, such as granular magnetic recording media, have rapidly developed over the last 20 years. The important key of these technologies was understanding magnetization structure and

${ }^{*}$ Corresponding author's email: dede.djuhana@sci.ui.ac.id, Tel.: +62-21-78849008 doi: 10.14716/ijtech.v12i3.4278 
reversal for individual grains or elements (Ali et al., 2018; Mu et al., 2019).

Recently, investigation of three-dimensional magnetic nanostructures has attracted much attention due to fundamental interest in magnetic properties as well as possible magnetic device applications (Manke et al., 2010; Streubel et al., 2015; Nur Fitriana et al., 2017; Sanz-Hernández et al., 2017; Suzuki et al., 2018; Fischer et al., 2020). In particular, micromagnetic simulations for three-dimensional magnetic nanostructures have been adopted to explain experimental results, in which the detailed domain structures at various magnetic states can be visualized (Aharoni, 2001; Fidler et al., 2002; Evans et al., 2014; Vousden et al., 2016; Leliaert and Mulkers, 2019). Micromagnetic simulation is a mezoscopic scale modeling, which can be solved using a finite difference or finite element discretization approach (Miltat and Donahue, 2007; Schrefl et al., 2007; Haryanto et al., 2017). Numerous studies have been reported that have investigated the domain structure in cube (Hertel and Kronmüller, 1999; Hertel and Kronmüller, 2002; Lu and Leonard, 2002; Piao et al., 2010), sphere (Boardman et al., 2004; Kákay and Varga, 2005; Yani et al., 2018; Usov and Nesmeyanov, 2020), cylinder (Piao et al., 2013; Fernandez-Roldan et al., 2019), and pyramidal shapes (Knittel et al., 2010). Among these, the case of nanospheres has steadily gained interest since it gives exciting features such as transitional domain structure from single-domain (SD) to multi-domain (MD), the critical size effect, and the possible magnetic vortex structure in spheres (Russier, 2009; Kurniawan et al., 2020). However, the ground state condition of a spherical-shaped magnetic nanoparticle around the domain structure transition with visual magnetization observation was rarely been studied.

In this work, micromagnetic simulation was utilized to explore the domain structure transition in ferromagnetic nanospheres at the ground-state conditions without an external magnetic field. The ferromagnetic materials consisting of $\mathrm{Ni}, \mathrm{Ni}_{0.8} \mathrm{Fe}_{0.2}$ (Py), $\mathrm{Fe}$, and Co have been investigated with the variation in diameters ranging from 20 to $100 \mathrm{~nm}$. A transitional behavior of the domain structure from SD to MD has been observed with critical conditions. The vortex structure was formed in an MD state with the vortex-core orientation toward the crystalline easy and hard axes of the materials. We have also calculated a critical diameter based on the analysis of magnetization energy around the transition with a comparable result to the theoretical Kittel and Brown equations.

\section{Methods}

The micromagnetic simulation was performed by a public micromagnetic solver program, an Object-Oriented Micromagnetic Framework (OOMMF) (Donahue and Porter, 1999), which is based on the Landau-Lifshitz-Gilbert (LLG) equation (Gilbert, 2004). The OOMMF used the finite difference approximation to solve the micromagnetic problems (Miltat and Donahue, 2007). The simulation process can be explained by minimizing the Gibbs free energy under the influence of a particular intrinsic effective field $\left(H_{\text {eff }}\right)$, composed of exchange, anisotropy, Zeeman, and demagnetization fields. Firstly, the sphere-shaped material model was initialized with specific material parameters and size. In this case, a nanosphere system with material parameters of $\mathrm{Co}, \mathrm{Fe}, \mathrm{Ni}$, and permalloy (Py) was explored with varying diameters ranging from 20 to $100 \mathrm{~nm}$. Table 1 presents the material parameters used in the simulation model (Skomski, 2001; López-Urías et al., 2005; Coey 2007): saturation magnetization $\left(M_{s}\right)$, exchange stiffness $(A)$, magnetic anisotropy constant $(K)$, and exchange length $\left(l_{\mathrm{ex}}\right)$. The simulation cell size was set to be $2.5 \times 2.5 \times 2.5 \mathrm{~nm}^{3}$, and the LLG damping factor was $\alpha=0.05$ for all materials. The exchange length was calculated from the equation $l_{e x}=\sqrt{2 A / \mu_{0} M_{S}^{2}}$ (Skomski, 2003; Coey, 2007; Getzlaff, 2008; Vaz et al., 2008; Guimarães, 2009). 
Table 1 The material parameters and the exchange lengths of $\mathrm{Co}, \mathrm{Fe}, \mathrm{Ni}$, and $\mathrm{Py}$ nanospheres with diameters from 20 to $100 \mathrm{~nm}$

\begin{tabular}{ccccc}
\hline Material & $\begin{array}{c}\text { Magnetization } \\
\text { Saturation, } M_{\mathrm{s}} \\
(\mathrm{A} / \mathrm{m}) \times 10^{5}\end{array}$ & $\begin{array}{c}\text { Exchange } \\
\text { Stiffness, } A \\
(\mathrm{~J} / \mathrm{m}) \times 10^{-12}\end{array}$ & $\begin{array}{c}\text { Anisotropy } \\
\text { Constant, } K \\
\left(\mathrm{~J} / \mathrm{m}^{3}\right) \times 10^{3}\end{array}$ & $\begin{array}{c}\text { Exchange } \\
\text { Length, lex } \\
(\mathrm{nm})\end{array}$ \\
\hline $\mathrm{Ni}$ & 4.9 & 9 & -5.7 & 7.72 \\
$\mathrm{Py}$ & 8.6 & 13 & 0.1 & 5.29 \\
$\mathrm{Fe}$ & 17 & 21 & 48 & 3.40 \\
$\mathrm{Co}$ & 14 & 30 & 530 & 4.93 \\
\hline
\end{tabular}

The initial magnetization was set to have a random spin configuration, as illustrated in Figure 1. Then, the simulation system was set to be relaxed without any external field or current until the magnetization energy reached equilibrium. Magnetization spin configuration in the three-dimensional sphere was presented and analyzed by converting the OOMMF Vector Field (OVF) data to the Visualisation Tool Kit (VTK) data by the OVF2VTK program (Fangohr, 2009).

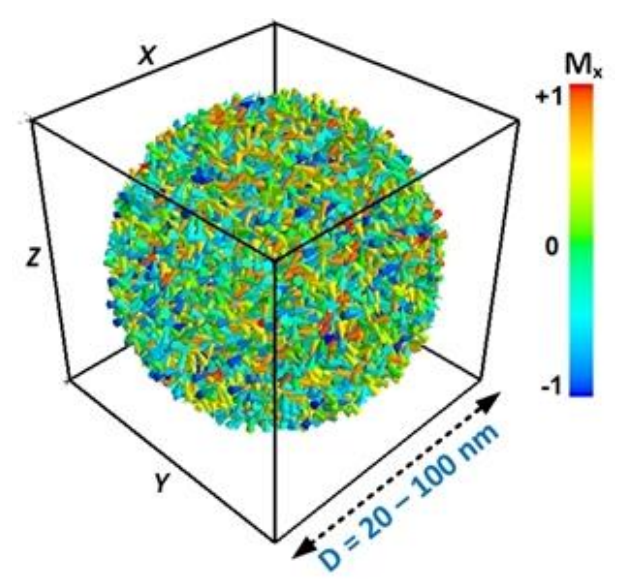

Figure 1 Initial magnetization state with random spin configuration. The color bar represents the magnetization direction

Furthermore, the critical diameter size of the domain structure transition from SD to MD was also analyzed. We used the Kittel and Brown equations to calculate the sphere model's theoretical critical transition diameter and compared it with the simulation results. The critical diameter equation as proposed by Kittel (Kittel, 1949) is given by

$$
D_{K}=\frac{72 \sqrt{A K}}{\mu_{0} M_{s}^{2}}
$$

Brown Jr. also proposed the critical diameter of ferromagnetic particles in two types (Brown, 1968). For the first type, the upper limit for the diameter of SD is given by

$$
D_{B 0}=7.211 \sqrt{\frac{2 A}{\mu_{0} M_{s}^{2}}}=7.211 l_{e x}
$$

For the second type, the diameter for the transition from SD to MD is given by

$$
D_{B 1}=\frac{9.0584 \sqrt{2 A / \mu_{0} M_{s}^{2}}}{1-2.8075\left(2 K / \mu_{0} M_{s}^{2}\right)}=\frac{9.0584}{1-2.8075 \kappa} l_{e x} \text { with } \quad \kappa=\frac{2 K}{\mu_{0} M_{s}^{2}}
$$




\section{Results and Discussion}

Under the minimum energy condition or equilibrium, a transition of domain structure from a single-domain structure (SD) to a multi-domain structure (MD) is found. The domain structure of SD exhibits magnetic configuration distribution in one direction, while the MD's domain structure tends to form a vortex-wall (VW). Figure 2 shows the magnetization energy with respect to the diameter variation for all materials with corresponding domain structures around the transition. It has been observed that the demagnetization and the exchange energies can be indicators that identify the transition of the domain structure. The demagnetization energy is mainly related to the magnetostatics mechanism, while the exchange energy is mostly contributed from the vortex structure (Kákay and Varga, 2005; Allenspach, 2007; Donahue, 2012; Betto and Coey, 2014; Kafrouni and Savadogo, 2016). Interestingly, the transition regime can be identified by the demagnetization energy decrease while the exchange energy increases (the dashed line in Figure 2). The region to the left of the dashed line in the domain structure shows the SD structure. The diameter value at the dashed line was $57.5,37.5,25$, and $50 \mathrm{~nm}$ for $\mathrm{Ni}, \mathrm{Py}, \mathrm{Fe}$, and Co, respectively. To the right of the dashed line, the domain structure exhibited MD structure. The MD structures emerge for the $\mathrm{Ni}, \mathrm{Py}, \mathrm{Fe}$, and Co spheres at a diameter of 60, 40, 27, and 52.5 $\mathrm{nm}$, respectively. It was confirmed that the application of the cell size around the exchange length of an individual material was sufficient for a stable investigation the transitional behavior of domain structure in the present study. It seems that our results of the magnetization energies profile and VW structure in the ferromagnetic spheres depicted the same trend as reported by Kákay's works (Kákay and Varga, 2005).

Next, we have examined the VW-core orientation in MD structure. Figure 3a shows Ni, $\mathrm{Py}, \mathrm{Fe}$, and Co spheres in the MD structure. For Ni, Fe, and Py spheres, the VW-core orientation exhibited two types: easy-axis orientation (EAO) and hard-axis orientation (HAO). For Co spheres, the VW-core orientation was observed only in HAO. Understanding the VW-core orientation in EAO and HAO can be considered by the crystal plane direction (Coey, 2007; Getzlaff, 2008). More interestingly, it is found that the VW-core orientation related to the crystal plane direction depended on the intrinsic properties such as anisotropy direction. This result confirms the VW orientation of Ni and Py spheres, that the EAO was in the direction [111] and the HAO was in the directions [100], [010], and [001]. For $\mathrm{Ni}$, the $\mathrm{HAO}$ was from 60 to $75 \mathrm{~nm}$, and the EAO was from 77.5 to $100 \mathrm{~nm}$. For Py, the HAO was from 40 to $42.5 \mathrm{~nm}$, and the EAO was from 45 to $100 \mathrm{~nm}$. The VW-core orientation of Fe spheres was oppositely directed from Ni and Py. The EAO was in the directions [100], [010], and [100] and the HAO was in the direction [111]. The evaluation shows the HAO was from 27.5 to $47.5 \mathrm{~nm}$, and the EAO was from 50 to $100 \mathrm{~nm}$. Contrary to the other cases, for the case of Co spheres, the VW-core orientation was observed only in one direction in the HAO since the EAO [0001] occurred in the SD structure. The HAO appeared from 52.5 to $100 \mathrm{~nm}$.

For further understanding, Figure $3 \mathrm{~b}$ presents the range HAO and EAO for all the materials. From the results for Ni, Py, and Fe spheres, it is observed that the VW-core orientation first occurred in $\mathrm{HAO}$ for a specific diameter range, and then stably changed to the EAO direction. In contrast, Co spheres showed only the VW-core orientation in HAO after the transition from SD. In this sense, the VW-core orientation transition from HAO to EAO is related to the minimization energy, where the minimization of the demagnetization energy will be maintained. In addition, HAO and EAO became important in the MD regime by applying the cell size around the exchange length and the small damping factor. 

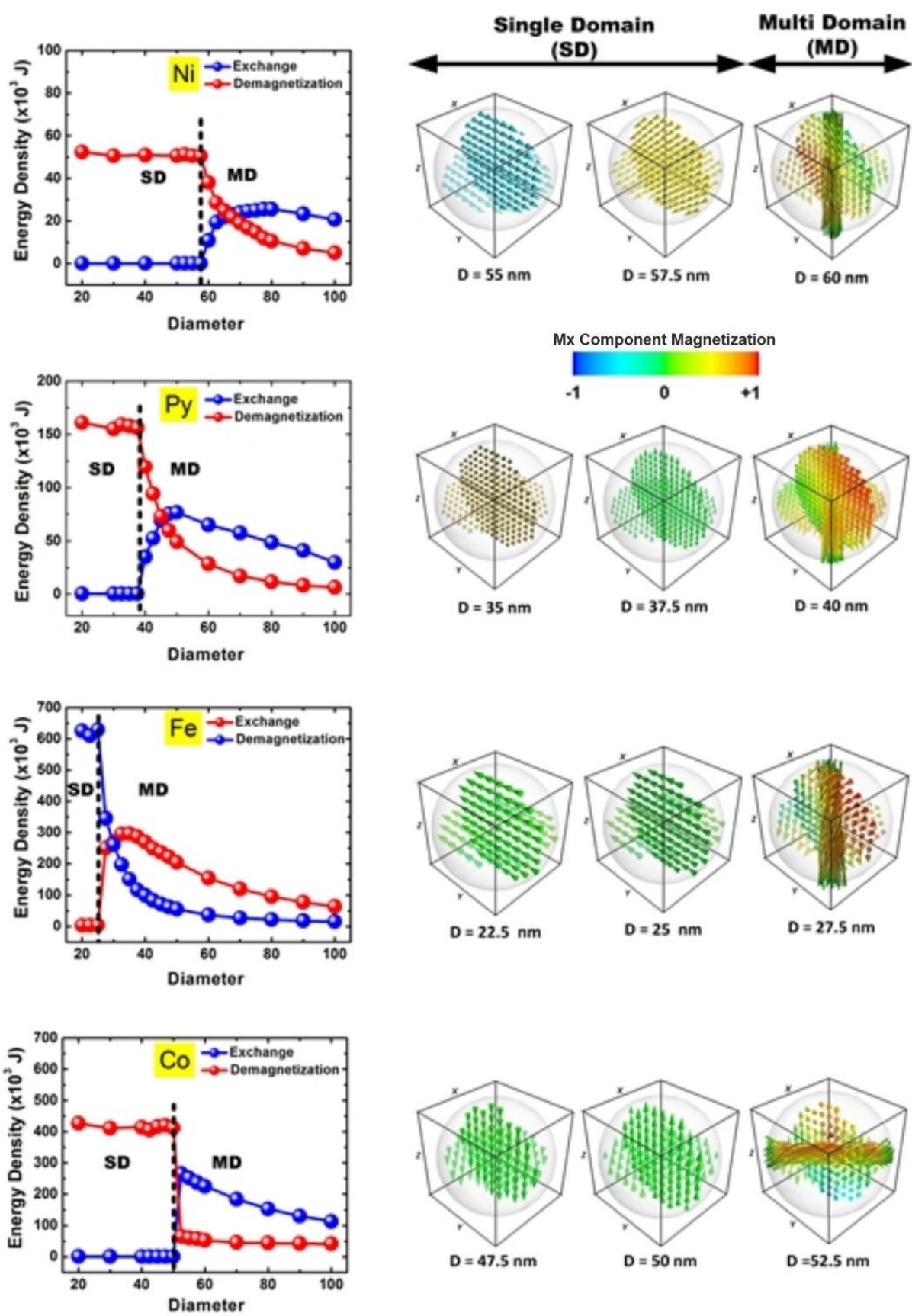

Figure 2 The demagnetization and exchange energy density profiles for $\mathrm{Ni}, \mathrm{Py}, \mathrm{Fe}$, and Co with respect to the sphere diameter. The dashed line denotes the domain transition region from SD to MD, where the demagnetization energy decreases and the exchange energy increases. The domain structures of $\mathrm{Ni}, \mathrm{Py}, \mathrm{Fe}$, and Co ferromagnets from SD to MD based on the energy density observation. The bar color represents the magnetization of the $M_{\mathrm{x}}$ component

Furthermore, the transition diameter of domain structure from SD to MD, called the critical diameter, for all material parameters was also analyzed by comparing the theoretical approach and simulation results. As mentioned in the previous section, the critical size of domain transition from SD to MD can be calculated by the Kittel (Kittel, 1949) and Brown equations (Brown, 1968). Table 2 shows the results of both the theoretical and micromagnetic calculations. 


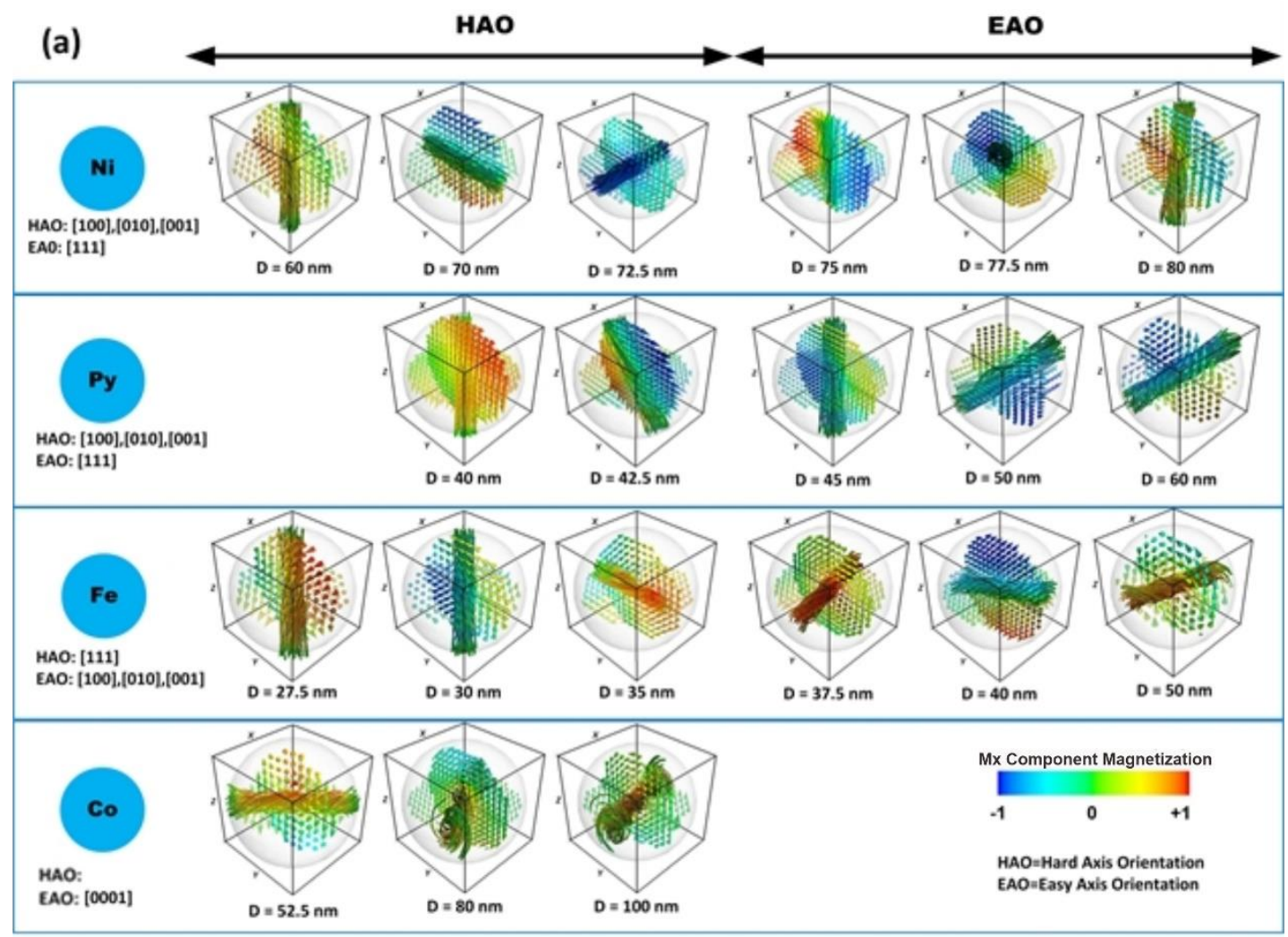

(b)

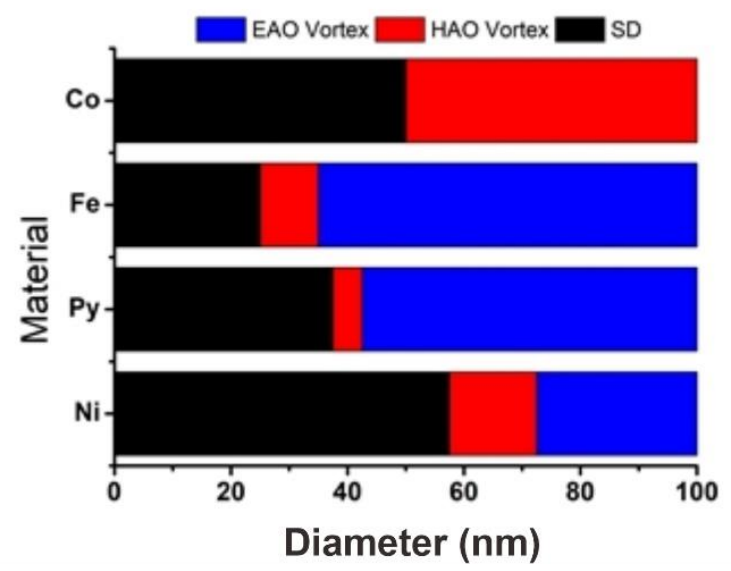

Figure 3 (a) The VW-core orientation of $\mathrm{Ni}, \mathrm{Py}, \mathrm{Fe}$, and Co spheres in MD structures. There are two types of VW-core orientation, easy-axis orientation (EAO) and hard-axis orientation (HAO). For the VW orientation of $\mathrm{Ni}$ and Py spheres, EAO is in [111], and HAO is in [100], [010], [001]. For the Fe sphere, EAO is in [100], [010],[001] and HAO is in [111]. The Co sphere has only HAO since EAO occurs in the SD structure. The color bar is the magnetization of the $M_{\mathrm{x}}$ component; (b) The range of $\mathrm{HAO}$ and EAO of the VW orientation for all materials

The critical diameter values of $\mathrm{Ni}$ and Fe spheres from micromagnetic simulation were found to be close to both Kittel $\left(D_{\mathrm{K}}\right)$ and the first type of Brown equation $\left(D_{\mathrm{B} 0}\right)$. Py spheres seem to preferentially follow the first type of Brown equation $\left(D_{\mathrm{B} 0}\right)$, while the Co spheres tend to conform to the second type of Brown equation $\left(D_{\mathrm{B} 1}\right)$. The critical diameter for lowanisotropy materials such as $\mathrm{Ni}, \mathrm{Py}$, and Fe seems to follow the Kittel equation and the first type of Brown equation. On the other hand, a high-anisotropy material such as Co follows the second type of Brown equation. As shown above, micromagnetic simulation is very useful in observing the transition of domain structure from SD to MD and the VW-core orientation with predicting the critical diameter. The value of the critical diameter also 
depends on the micromagnetic shapes and the simulation cell model. In this case, the micromagnetic simulation used the finite difference method with the rectangular cells. The cell size is relatively small for reducing the stair-step effect on the surface (Donahue and McMichael, 2007). Therefore, the interpretation of these simulation results was important for understanding the intrinsic behavior of favored magnetization orientation in the nanospherical magnetic materials. This work could be an important step in the material selection for magnetic granular-based storage.

Table 2 The critical diameter calculation based on Kittel (Kittel, 1949) and Brown equations (Brown, 1968) and micromagnetic simulation

\begin{tabular}{cccccc}
\hline \multirow{2}{*}{ Material } & \multicolumn{3}{c}{ Theoretical } & \multicolumn{2}{c}{ Micromagnetic } \\
& $D_{\mathrm{K}}(\mathrm{nm})$ & $D_{\mathrm{B} 0}(\mathrm{~nm})$ & $D_{\mathrm{B} 1}(\mathrm{~nm})$ & $D_{\mathrm{SD}}(\mathrm{nm})$ & $D_{\mathrm{MD}}(\mathrm{nm})$ \\
\hline $\mathrm{Ni}$ & 54.05 & 55.70 & 78.27 & 57.5 & 60.0 \\
$\mathrm{Py}$ & 2.79 & 38.14 & 47.94 & 37.5 & 40.0 \\
$\mathrm{Fe}$ & 19.90 & 24.52 & 33.27 & 25.0 & 27.5 \\
$\mathrm{Co}$ & 36.86 & 35.59 & 50.85 & 50.0 & 52.5 \\
\hline
\end{tabular}

\section{Conclusions}

In conclusion, we have systematically observed the transition of the domain structure of $\mathrm{Ni}, \mathrm{Py}, \mathrm{Fe}$, and Co sphere models using micromagnetic simulation at groundstate conditions without an external magnetic field. The transition domain structure from SD to MD was analyzed based on the magnetization energies, namely the demagnetization and exchange energy. The $\mathrm{MD}$ is first recognized when the demagnetization energy decreases while the exchange energy increases. A VW structure is formed in the MD regime, and the core orientation of the VW structure has two orientations, HAO and EAO. It is found that the HAO and EAO of the VW structure relate to the crystal plane direction. The critical diameter at the transition from SD to MD was also determined. Interestingly, the simulation results show good agreement compared with the theoretical Kittel and Brown equations. Therefore, an interpretation of the magnetization dynamic is an important step in the material selection for magnetic granular-based storage.

\section{Acknowledgements}

This work is fully supported by Hibah Penelitian Dasar Unggulan Perguruan Tinggi (PDUPT) year 2020 from the Ministry of Research, Technology, and Higher Education of the Republic of Indonesia with the contract number NKB-202/UN2.RST/HKP.05.00/2020. We also thank DRPM Universitas Indonesia for facilitating this research.

\section{References}

Aharoni, A., 2001. Micromagnetics: Past, Present and Future. Physica B: Condensed Matter, Volume 306(1-4), pp. 1-9

Ali, S.R., Naz, F., Akber, H., Naeem, M., Ali, S.I., Basit, S.A., Sarim, M., Qaseem, S., 2018. Effect of Particle Size Distribution on Magnetic Behavior of Nanoparticles with Uniaxial Anisotropy. Chinese Physics B, Volume 27(9), 097503-1-097503-5

Allenspach, R., 2007. Magnetic Properties of Systems of Low Dimensions. Handbook of Magnetism and Advanced Magnetic Materials, Kronmüller, H., Parkin, S. (Eds.), John Wiley \& Sons, Ltd, Chichester, UK 
Betto, D., Coey, J.M.D., 2014. Vortex State in Ferromagnetic Nanoparticles. Journal of Applied Physics, Volume 115(17), 17D138, https://doi.org/10.1063/1.4867597

Bhatti, S., Sbiaa, R., Hirohata, A., Ohno, H., Fukami, S., Piramanayagam, S.N., 2017. Spintronics Based Random Access Memory: A Review. Materials Today, Volume 20(9), pp. $530-548$

Boardman, R.P., Fangohr, H., Cox, S.J., Goncharov, A.V., Zhukov, A.A., de Groot, P.A.J., 2004. Micromagnetic Simulation of Ferromagnetic Part-Spherical Particles. Journal of Applied Physics, Volume 95(11), pp. 7037-7039

Brown, W.F., 1968. The Fundamental Theorem of Fine-Ferromagnetic-Particle Theory. Journal of Applied Physics, Volume 39, pp. 993-994

Coey, J.M.D., 2007. Magnetism and Magnetic Materials. Cambridge University Press

Donahue, M.J., 2012. Micromagnetic Investigation of Periodic Cross-Tie/Vortex Wall Geometry. Advances in Condensed Matter Physics, Volume 2012, pp. 1-8

Donahue, M.J., McMichael, R.D., 2007. Micromagnetics on Curved Geometries using Rectangular Cells: Error Correction and Analysis. IEEE Transactions on Magnetics, Volume 43(6), pp. 2878-2880

Donahue, M.J., Porter, D.G., 1999. OOMMF User's Guide, Version 1.0, Interagency Report NISTIR 6376. National Institute of Standards and Technology, Gaithersburg, MD

Evans, R.F.L., Fan, W.J., Chureemart, P., Ostler, T.A., Ellis, M.O.A., Chantrell, R.W., 2014. Atomistic Spin Model Simulations of Magnetic Nanomaterials. Journal of Physics: Condensed Matter, Volume 26(10), pp. 1-23

Fangohr, H., 2009. OVF2VTK: Tool for Conversion of OOMMF to VTK Files [WWW Document]. Available Online at https://fangohr.github.io/software/ovf2vtk/index.html

Fernandez-Roldan, J.A., De Riz, A., Trapp, B., Thirion, C., Vazquez, M., Toussaint, J.-C., Fruchart, O., Gusakova, D., 2019. Modeling Magnetic-Field-Induced Domain Wall Propagation in Modulated-Diameter Cylindrical Nanowires. Scientific Reports, Volume 9(5130), pp. 1-12

Fidler, J., Schrefl, T., Tsiantos, V.D., Scholz, W., Suess, D., 2002. Micromagnetic Simulation of the Magnetic Switching Behaviour of Mesoscopic and Nanoscopic Structures. Computational Materials Science, Volume 24(1-2), pp. 163-174

Fischer, P., Sanz-Hernández, D., Streubel, R., Fernández-Pacheco, A., 2020. Launching a New Dimension with 3D Magnetic Nanostructures. APL Materials, Volume 8, pp. 010701-1 - 010701-12

Getzlaff, M., 2008. Fundamentals of Magnetism. New York: Springer Science \& Business Media

Gilbert, T.L., 2004. Classics in Magnetics: A Phenomenological Theory of Damping in Ferromagnetic Materials. IEEE Transactions on Magnetics, Volume 40(6), pp. 34433449

Guimarães, A.P., 2009. Principles of Nanomagnetism, NanoScience and Technology. Berlin Heidelberg: Springer

Haryanto, Y., Gan, B.S., Maryoto, A., 2017. Wire Rope Flexural Bonded Strengthening System on RC-Beams: A Finite Element Simulation. International Journal of Technology, Volume 8(1), pp. 132-144

Hertel, R., Kronmüller, H., 2002. Finite Element Calculations on the Single-Domain Limit of a Ferromagnetic Cube: A Solution to $\mu \mathrm{MAG}$ Standard Problem No. 3. Journal of Magnetism and Magnetic Materials, Volume 238(2-3), pp. 185-199

Hertel, R., Kronmüller, H., 1999. Computation of the Magnetic Domain Structure in Bulk Permalloy. Physical Review B, Volume 60(10), pp. 7366-7378 
Joshi, V.K., 2016. Spintronics: A Contemporary Review of Emerging Electronics Devices. Engineering Science and Technology, an International Journal, Volume 19(3), pp. 15031513

Kafrouni, L., Savadogo, O., 2016. Recent Progress on Magnetic Nanoparticles for Magnetic Hyperthermia. Progress in Biomaterials, Volume 5, pp. 147-160

Kákay, A., Varga, L.K., 2005. Monodomain Critical Radius for Soft-Magnetic Fine Particles. Journal of Applied Physics, Volume 97, 083901, https://doi.org/10.1063/1.1844612

Kittel, C., 1949. Physical Theory of Ferromagnetic Domains. Reviews of Modern Physics, Volume 21, pp. 541-583

Knittel, A., Franchin, M., Fischbacher, T., Nasirpouri, F., Bending, S.J., Fangohr, H., 2010. Micromagnetic Studies of Three-Dimensional Pyramidal Shell Structures. New Journal of Physics, Volume 12, pp. 1-23

Kurniawan, C., Widodo, A.T., Kim, D.H., Djuhana, D., 2020. Micromagnetic Investigation of Magnetization Reversal in Sphere-Shaped Ferromagnetic Nanoparticle. Key Engineering Materials, Volume 855, pp. 237-242

Leliaert, J., Mulkers, J., 2019. Tomorrow's Micromagnetic Simulations. Journal of Applied Physics, Volume 125, pp. 180901-1- 180901-9

López-Urías, F., Torres-Heredia, J.J., Muñoz-Sandoval, E., 2005. Magnetization Patterns Simulations of $\mathrm{Fe}, \mathrm{Ni}, \mathrm{Co}$, and Permalloy Individual Nanomagnets. Journal of Magnetism and Magnetic Materials, Volume 294(2), pp. e7-e12

Lu, M., Leonard, P.J., 2002. Micromagnetic Simulation of Magnetization Reversal Processes in Ferromagnetic Cubes from Quasisaturation State. Journal of Physics: Condensed Matter, Volume 14, pp. 8089-8101

Manke, I., Kardjilov, N., Schäfer, R., Hilger, A., Strobl, M., Dawson, M., Grünzweig, C., Behr, G., Hentschel, M., David, C., Kupsch, A., Lange, A., Banhart, J., 2010. Three-Dimensional Imaging of Magnetic Domains. Nature Communications, Volume 1(125), pp. 1-6

Miltat, J.E., Donahue, M.J., 2007. Numerical Micromagnetics: Finite Difference Methods. Handbook of Magnetism and Advanced Magnetic Materials, Helmut Kronmuller and Stuart Parkin (eds.), Volume 2: Micromagnetism, John Wiley \& Sons, Ltd., pp. 1-23

$\mathrm{Mu}, \mathrm{C}$. Jing, J., Dong, J., Wang, W., Xu, J., Nie, A., Xiang, J., Wen, F., Liu, Z., 2019. Static and Dynamic Characteristics of Magnetism in Permalloy Oval Nanoring by Micromagnetic Simulation. Journal of Magnetism and Magnetic Materials, Volume 474, pp. 301-304

Nur Fitriana, K., Hafizah, M.A.E., Manaf, A., 2017. Synthesis and Magnetic Characterization of Mn-Ti Substituted SrO.6Fe2-xMn $/ 2 \mathrm{Ti}_{x} / 2 \mathrm{O}_{3}(\mathrm{x}=0.0-1.0)$ Nanoparticles by Combined Destruction Process. International Journal of Technology., Volume 8(4), pp. 644-650

Piao, H.-G., Djuhana, D., Shim, J.-H., Lee, S.-H., Jun, S.-H., Oh, S.K., Yu, S.-C., Kim, D.-H., 2010. Three-Dimensional Spin Configuration of Ferromagnetic Nanocubes. Journal of Nanoscience and Nanotechnology, Volume 10(11), pp. 7212-7216

Piao, H.-G., Shim, J.-H., Djuhana, D., Kim, D.-H., 2013. Intrinsic Pinning Behavior and Propagation Onset of Three-Dimensional Bloch-Point Domain Wall in a Cylindrical Ferromagnetic Nanowire. Applied Physics Letters, Volume 102(11), 112405, https://doi.org/10.1063/1.4794823

Russier, V., 2009. Spherical Magnetic Nanoparticles: Magnetic Structure and Interparticle Interaction. Journal of Applied Physics, Volume 105(7), 073915, https://doi.org/10.1063/1.3093966

Sanz-Hernández, D., Hamans, R.F., Liao, J.-W., Welbourne, A., Lavrijsen, R., FernándezPacheco, A., 2017. Fabrication, Detection, and Operation of a Three-Dimensional Nanomagnetic Conduit. ACS Nano, Volume 11(11), pp. 11066-11073 
Sbiaa, R., Piramanayagam, S.N., 2017. Recent Developments in Spin Transfer Torque MRAM. Physica Status Solidi RRL - Rapid Research Letters, Volume 11(12), 1700163, https://doi.org/10.1002/pssr.201700163

Schrefl, T., Hrkac, G., Bance, S., Suess, D., Ertl, O., Fidler, J., 2007. Numerical Methods in Micromagnetics (Finite Element Method). In: Handbook of Magnetism and Advanced Magnetic Materials, Kronmüller, H., Parkin, S. (Eds.), John Wiley \& Sons, Ltd, Chichester, UK, p. hmm203

Skomski, R., 2001. Micromagnetic Spin Structure. In: Spin Electronics, Lecture Notes in Physics, Ziese, M., Thornton, M.J. (Eds.), Springer Berlin Heidelberg, Berlin, Heidelberg, pp. 204-231

Skomski, R., 2003. Nanomagnetics. Journal of Physics: Condensed Matter, Volume 15(20), pp. R841-R896

Streubel, R., Kronast, F., Fischer, P., Parkinson, D., Schmidt, O.G., Makarov, D., 2015. Retrieving Spin Textures on Curved Magnetic Thin Films with Full-Field Soft X-ray Microscopies. Nature Communications, Volume 6(7612), pp. 1-11

Sun, L., Wong, P.K.J., Zhang, W., Zhai, Y., Niu, D.X., Xu, Y.B., Zou, X., Zhai, H.R., 2015. Micromagnetic Simulation on the Interelement Coupling of High-Density Patterned Film. IEEE Transactions on Magnetics, Volume 51(11), pp. 1-4

Suzuki, M., Kim, K.-J., Kim, S., Yoshikawa, H., Tono, T., Yamada, K.T., Taniguchi, T., Mizuno, H., Oda, K., Ishibashi, M., Hirata, Y., Li, T., Tsukamoto, A., Chiba, D., Ono, T., 2018. ThreeDimensional Visualization of Magnetic Domain Structure with Strong Uniaxial Anisotropy Via Scanning Hard X-ray Microtomography. Applied Physics Express, Volume 11(3), pp. 036601-1-036601-4

Udhiarto, A., Tamam, M.A., Nuryadi, R., Hartanto, D., 2014. Design and Simulation of Two Bits Single-Electron Logic Circuit using Double Quantum Dot Single Electron Transistor. International Journal of Technology, Volume 5(2), pp. 152-158

Usov, N.A., Nesmeyanov, M.S., 2020. Multi-Domain Structures in Spheroidal Co Nanoparticles. Scientific Reports, Volume 10(10173), pp. 1-9

Vaz, C.A.F., Bland, J.A.C., Lauhoff, G., 2008. Magnetism in Ultrathin Film Structures. Reports on Progress in Physics, Volume 71(5), pp. 1-78

Vousden, M., Bisotti, M.-A., Albert, M., Fangohr, H., 2016. Virtual Micromagnetics: A Framework for Accessible and Reproducible Micromagnetic Simulation. Journal of Open Research Software, Volume 4(1), pp. 1-7

Yani, A., Kurniawan, C., Djuhana, D., 2018. Investigation of the Ground State Domain Structure Transition on Magnetite $\left(\mathrm{Fe}_{3} \mathrm{O}_{4}\right)$. In: Proceedings of the $3^{\text {rd }}$ International Symposium on Current Progress in Mathematics and Sciences 2017 (ISCPMS2017), Bali, Indonesia, p. 020020. 